\title{
MiR-194 functions as a tumor suppressor in laryngeal squamous cell carcinoma by targeting Wee1
}

\author{
Pei $\mathrm{Li}^{1+}$, Yang Yang ${ }^{2+}$, Hui Liu ${ }^{3+}$, An-Kui Yang ${ }^{4}$, Jin-Ming Di ${ }^{5}$, Guang-Mou Tan ${ }^{6}$, Hai-Feng Wang ${ }^{1}$, Jian-Ge Qiu², \\ Wen-Ji Zhang ${ }^{2}$, Qi-Wei Jiang ${ }^{2}$, Di-Wei Zheng ${ }^{2}$, Yao Chen², Meng-Ning Wei ${ }^{2}$, Jia-Rong Huang ${ }^{2}$, Kun Wang ${ }^{2}$, \\ Zhi Shi ${ }^{2^{*}}$ (D) and Jin Ye 1* $^{\text {}^{*}}$
}

\begin{abstract}
The emerging roles of microRNAs (miRs) have been deeply investigated in cancer. However, the role of miR-194 in human laryngeal squamous cell carcinoma (LSCC) is still unclear. Here, we have demonstrated that miR-194 is significantly downregulated in LSCC tissues and cells, and overexpression of miR-194 inhibits the proliferation, migration, invasion, and drug resistance in LSCC cells. Moreover, Wee1 is identified as a novel direct target of miR-194. Ectopic expression of Wee1 at least in part overcomes the suppressive impacts of miR-194 on the malignant phenotypes of LSCC. Overall, our study provides new sights into the role of miR-194/Wee1 axis in LSCC and suggests a novel miR-194/Wee1-based clinical application for LSCC patients.
\end{abstract}

Keywords: Laryngeal squamous cell carcinoma, miR-194, Wee1

\section{Letter to the editor}

Laryngeal cancer is the fourteenth most prevalent type of malignancy worldwide in the male compared to its relative rare in the female. Laryngeal squamous cell carcinoma (LSCC) accounts for approximately $90 \%$ of all malignant tumors of the larynx [1]. Deregulated microRNAs (miRs) are frequently demonstrated as biomarker or therapeutic target in LSCC tissues and cells, which may function as tumor suppressor or oncogene to regulate the malignancy of cancer [2]. As one of frequently deregulated miRs in cancer, the expression and role of miR-194 in LSCC are still unknown [3, 4].

In the current study, we found that the expression level of miR-194 is significantly lower not only in two LSCC cell lines Hep-2 and KB-3-1 compared with

\footnotetext{
* Correspondence: tshizhi@jnu.edu.cn; yejin_sums@aliyun.com

${ }^{\dagger}$ Equal contributors

${ }^{2}$ Department of Cell Biology \& Institute of Biomedicine, National Engineering Research Center of Genetic Medicine, Guangdong Provincial Key Laboratory of Bioengineering Medicine, College of Life Science and Technology, Jinan University, Guangzhou, Guangdong, China

${ }^{1}$ Department of Otolaryngology-Head and Neck Surgery, The Third Affiliated Hospital, Sun Yat-sen University, Guangzhou, Guangdong, China

Full list of author information is available at the end of the article
}

normal human bronchial epithelial cell line 16HBE but also in clinical LSCC tissues compared with adjacent normal tissues and correlated with $\mathrm{T}$ stage, lymph node metastasis, and clinical stage, but not with age, tumor grades, and tumor primary locations (Fig. 1b-g, Additional file 1: Figure S1a and Table S1). The miR-194 levels could be a significant parameter to distinguish LSCC and adjacent normal tissues with an area under the ROC curve (AUC) of 0.676 $($ sensitivity $=63.64 \%, \quad$ specificity $=72.72 \% ; \quad P=0.004)$ (Fig. 1h). Kaplan-Meier analysis indicated that high miR-194 expression predicts a favorable outcome for LSCC patients (Fig. 1i). Functional assays showed that enforced expression of miR-194 inhibits the growth, migration, invasion, and drug resistance of Hep- 2 and KB3-1 cells in vitro (Additional file 1: Figure S2a-g). The data in the subcutaneous tumor model in nude mice revealed that overexpression of miR-194 significantly inhibited the growth of Hep-2 and KB-3-1 xenografts by the numbers of $\mathrm{Ki}^{+} 7^{+}$proliferating cells and $\mathrm{CD} 31^{+}$ microvessels (Additional file 1: Figure S3a-d). We used 


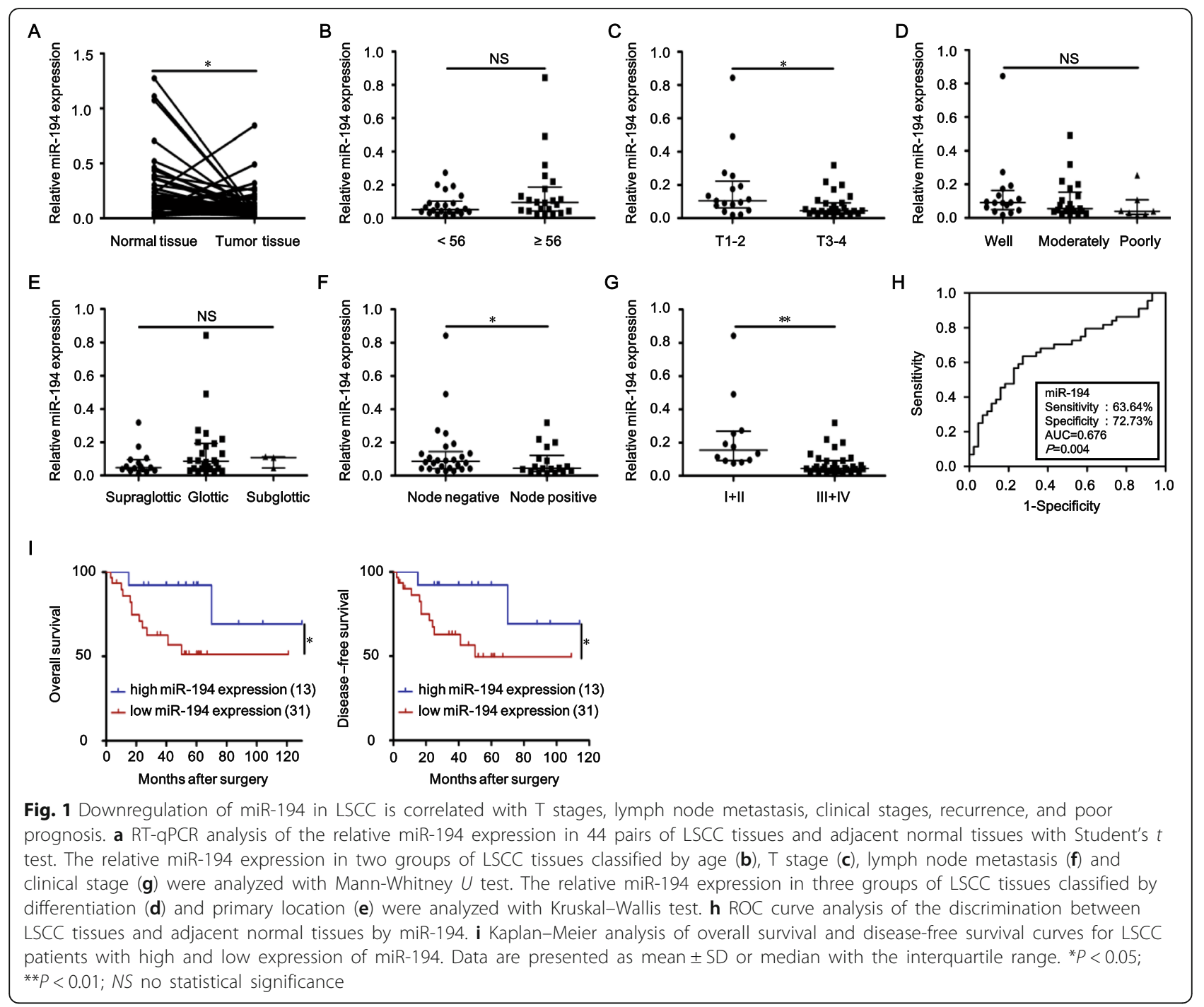

three computational algorithms, including miRanda, PITA, and TargetScan in combination to identify the novel potential targets of miR-194. All three algorithms predicted Wee1 as a target gene of miR-194, which is a protein kinase to regulate cell cycle through inhibiting CDK1 by phosphorylation on its two different residues Thr14 and Tyr15 [5]. Western blot analysis showed that ectopic expression of miR-194 in Hep-2 and KB-3-1 cells obviously downregulated the protein levels of Wee1 as well as the known targets XIAP and p27 [3, 6] (Fig. 2a). The predicted interaction between miR-194 and the target sites within the 3'-untranslated regions (3'UTR) of Wee1 was shown in Fig. 2b. We then performed luciferase assay to examine whether there is a direct interaction between miR-194 and Wee1. The wild type or mutant 3'UTR region of Wee1 were cloned into downstream of the firefly luciferase gene to generate the luciferase reporter vectors. As illustrated in Fig. 2c, ectopic expression of miR-194 significantly decreased the luciferase activity of wild type 3'UTR, but not mutant 3'UTR in Hep-2 and KB-3-1 cells, suggesting that the putative miRNA binding sites of Wee1 are responsible for this miRNA-mRNA interaction (Additional file 1: Figure S1b). Furthermore, analysis of proteins extracted from Hep-2 and KB-3-1 subcutaneous tumors in mice exhibited that the protein levels of Wee1 in miR-194-transduced tumors were remarkably lower than those in vector control (Fig. 2d). In addition, ectopic expression of Wee1 partially reverses the suppressive effects of miR-194 on LSCC cells (Additional file 1: Figure S4a-g).

In summary, our data reveal a potential suppressive role of miR-194 in LSCC by targeting Wee1 in vitro and in vivo. The clinical results indicate that miR-194 

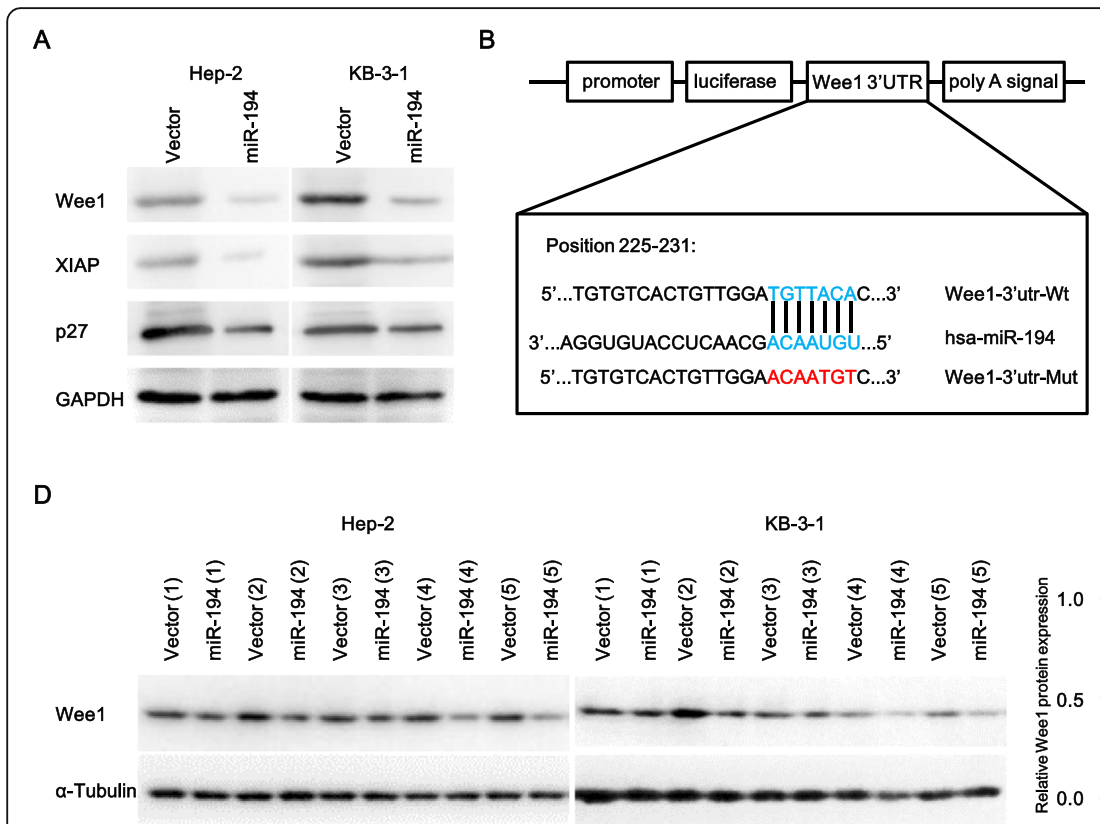

C

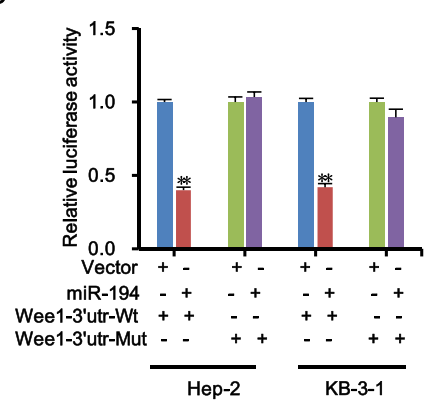

Fig. 2 MiR-194 directly targets Wee1. a Western blot analysis of Wee1, XIAP, and p27 protein expressions in the indicated cells. GAPDH is the loading control. $\mathbf{b}$ A schematic diagram of the reporter constructs showed the wild type (Wt) and mutant (Mut) sequences of the miR-194 binding sites within human Wee1 3'-UTR. c Luciferase activity of reporters with Wee1 Wt or Mut 3'-UTR in the indicated cells. d Western blot analysis and quantification of Wee1 protein expression in the tumors of the indicated cells. A-Tubulin was the loading control. Data are presented as mean \pm SD. Student's $t$ test was used for statistical analysis. ${ }^{* *} P<0.01$

can be the potential diagnostic and prognostic biomarkers for LSCC. Our study provides new sights into the role of miR-194/Wee1 axis in LSCC and suggests a novel miR-194/Wee1-based clinical application for LSCC patients.

\section{Additional file}

Additional file 1: Supplemental data. (PDF $1209 \mathrm{~kb}$ )

\section{Abbreviations}

LSCC: Laryngeal squamous cell carcinoma; UTRs: 3'-untranslated regions

\section{Acknowledgements}

We thank other members of Shi lab and Ye lab for their comments and critical reading of the manuscript.

\section{Funding}

This work was supported by funds from the Chinese National Natural Science Foundation Nos. 8161101021,31271444 and No. 81201726 (Z. S.), the Guangdong Natural Science Funds for Distinguished Young Scholar No. 2014 A030306001 (Z. S.) and No. 2014 A030313057 (J. Y.), the Guangdong Special Support Program for Young Talent No. 2015TQ01R350 (Z. S.), the Science and Technology Program of Guangdong No. 2016 A050502027 (Z. S.) and No. 2013 B021800088 (J. Y.), and the Foundation for Research Cultivation and Innovation of Jinan University No. 21616119 (Z.S.).

\section{Availability of data and materials}

The datasets supporting the conclusions of this article are included within the article and its additional files.

\section{Author's contributions}

$P L, Y Y, H L, Z S$, and JY designed the experiments, performed the experiments, analyzed the data, and wrote the paper. AKY, JMD, GMT, HFW, JGQ, WJZ, QWJ, DWZ, YC, MNW, JRH, and KW performed the experiments. All authors read and approved the final manuscript.

\section{Competing interests}

The authors declare that they have no competing interests.

\section{Consent for publication}

Not applicable.

\section{Ethics approval and consent to participate}

This project was approved by the Ethics Committee of Sun Yat-sen University Cancer Center. Ethics, consent, and permissions: Informed consent was obtained from each patient enrolled in the study. All animal experimental procedures were approved by the Institutional Animal Care and Use Committee of Sun Yat-Sen University.

\section{Author details}

'Department of Otolaryngology-Head and Neck Surgery, The Third Affiliated Hospital, Sun Yat-sen University, Guangzhou, Guangdong, China.

2Department of Cell Biology \& Institute of Biomedicine, National Engineering Research Center of Genetic Medicine, Guangdong Provincial Key Laboratory of Bioengineering Medicine, College of Life Science and Technology, Jinan University, Guangzhou, Guangdong, China. ${ }^{3}$ Division of Pulmonary and Critical Care, Department of Internal Medicine, The Third Affiliated Hospital, Sun Yat-sen University, Guangzhou, Guangdong, China. ${ }^{4}$ Department of Head and Neck, State Key Laboratory of Oncology in South China, Collaborative Innovation Center for Cancer Medicine, Sun Yat-sen University Cancer Center, Guangzhou, Guangdong, China. ${ }^{5}$ Department of Urology, The Third Affiliated Hospital, Sun Yat-sen University, Guangzhou, Guangdong, China. ${ }^{6}$ Department of Head \& Neck Surgery, Affiliated Cancer Hospital \& Institute of Guangzhou Medical University, Guangzhou, China.

Received: 24 December 2016 Accepted: 13 January 2017 Published online: 25 January 2017 


\section{References}

1. Siegel RL, Miller KD, Jemal A. Cancer statistics, 2015. CA Cancer J Clin. 2015;65:5-29.

2. Li P, Liu H, Wang ZY, He F, Wang HF, Shi Z, Yang AK, Ye J. MicroRNAs in laryngeal cancer: implications for diagnosis, prognosis and therapy. Am J Transl Res. 2016:8:1935-44.

3. Wu X, Liu T, Fang O, Leach $\sqcup$, Hu X, Luo Z. miR-194 suppresses metastasis of non-small cell lung cancer through regulating expression of BMP1 and p27(kip1). Oncogene. 2014;33:1506-14.

4. Zhang M, Zhuang Q, Cui L. MiR-194 inhibits cell proliferation and invasion via repression of RAP2B in bladder cancer. Biomed Pharmacother. 2016;80:268-75.

5. Matheson CJ, Backos DS, Reigan P. Targeting WEE1 Kinase in Cancer. Trends Pharmacol Sci. 2016;37:872-81.

6. Zhao HJ, Ren LL, Wang ZH, Sun TT, Yu YN, Wang YC, Yan TT, Zou W, He J, Zhang Y, Hong J, Fang JY. MiR-194 deregulation contributes to colorectal carcinogenesis via targeting AKT2 pathway. Theranostics. 2014;4:1193-208.

Submit your next manuscript to BioMed Central and we will help you at every step:

- We accept pre-submission inquiries

- Our selector tool helps you to find the most relevant journal

- We provide round the clock customer support

- Convenient online submission

- Thorough peer review

- Inclusion in PubMed and all major indexing services

- Maximum visibility for your research

Submit your manuscript at www.biomedcentral.com/submit
Biomed Central 